\title{
Criminologie
}

\section{Passer les titres en revue : contribution à l'histoire de Criminologie}

\section{Dan Kaminski}

Volume 41, numéro 1, printemps 2008

40 ans de Criminologie : perspectives d'avenir

URI : https://id.erudit.org/iderudit/018418ar

DOI : https://doi.org/10.7202/018418ar

Aller au sommaire du numéro

\section{Éditeur(s)}

Les Presses de l'Université de Montréal

ISSN

0316-0041 (imprimé)

1492-1367 (numérique)

Découvrir la revue

Citer cet article

Kaminski, D. (2008). Passer les titres en revue : contribution à l'histoire de Criminologie. Criminologie, 41(1), 17-46. https://doi.org/10.7202/018418ar
Résumé de l'article

Cet article se présente comme une contribution à l'histoire formelle de la revue Criminologie. Une histoire formelle signifie fondamentalement une histoire des signes et repose sur l'hypothèse selon laquelle les mots - plus spécifiquement les mots contribuant à la formation des titres des articles publiés - constituent des sources utilisables pour une connaissance diachronique de la discipline à laquelle ils appartiennent. Pour réaliser cette histoire, on s'est d'abord penché sur le catalogue de la revue, soit l'ensemble des titres publiés entre 1968 et 2005. Ce fichier est composé de 527 références comprenant des informations telles que l'auteur, le titre, l'année, le titre du numéro, la nationalité de l'auteur et la nature de la contribution. Une deuxième série d'analyses a porté sur les thèmes des livraisons, thèmes indiqués par le titre général donné à chaque numéro (67 numéros entre 1968 et 2005). Finalement, une analyse des éditoriaux de tous les numéros publiés pendant la même période complète les analyses. Cette recherche empirique permet non seulement de tracer les contours de la transformation de la revue Criminologie, mais plus largement ceux de la discipline. 


\title{
Passer les titres en revue: contribution à l'histoire de Criminologie
}

\author{
Dan Kaminski \\ Professeur \\ École de criminologie, Université catholique de Louvain \\ kaminski@crim.ucl.ac.be
}

\begin{abstract}
RÉSUMÉ • Cet article se présente comme une contribution à l'histoire formelle de la revue Criminologie. Une histoire formelle signifie fondamentalement une histoire des signes et repose sur l'hypothèse selon laquelle les mots - plus spécifiquement les mots contribuant à la formation des titres des articles publiés - constituent des sources utilisables pour une connaissance diachronique de la discipline à laquelle ils appartiennent. Pour réaliser cette histoire, on s'est d'abord penché sur le catalogue de la revue, soit l'ensemble des titres publiés entre 1968 et 2005 . Ce fichier est composé de 527 références comprenant des informations telles que l'auteur, le titre, l'année, le titre du numéro, la nationalité de l'auteur et la nature de la contribution. Une deuxième série d'analyses a porté sur les thèmes des livraisons, thèmes indiqués par le titre général donné à chaque numéro (67 numéros entre 1968 et 2005). Finalement, une analyse des éditoriaux de tous les numéros publiés pendant la même période complète les analyses. Cette recherche empirique permet non seulement de tracer les contours de la transformation de la revue Criminologie, mais plus largement ceux de la discipline.
\end{abstract}

ABSTRACT - This article is a contribution to the formal history of the journal Criminologie. A formal history implies a history of the signs. It rests upon the hypothesis according to which words - most especially those used in the title of the articles - are a valuable source to map the history of criminological discipline. In order to achieve this goal, we first scrutinized the journal catalogue. This file is made of the 527 references with such information as the authors' name, article's title, publication year, title of the issue, authors' nationality and type of article. The second series of analysis focused on the topic of the issues as indicated by their titles (67 issues between 1968 and 2005). Finally, a thorough analysis was performed on the editorials for each issue covering the whole period under study. This empirical research allows us to trace not only the evolution of the journal Criminologie but more broadly of the discipline it respresents. 


\section{Introduction}

L'exercice auquel je me suis astreint pour ce numéro jubilaire de Criminologie pourra sembler formel. En quelque sorte je revendique ce formalisme: j'ai tenté de réaliser une modeste contribution à l'histoire formelle de la revue en m'appuyant essentiellement sur des indices comptables ou empiriquement constatables de son évolution. Une histoire formelle signifie fondamentalement une histoire des signes, faisant abstraction des hommes et des femmes qui les ont tracés; une histoire qui fonde la vitalité propre d'une revue dans l'univers sans auteur du discours criminologique de son temps. Les noms des hommes et des femmes apparaîtront certes, mais aucune gloire ne leur sera attribuée; ces noms sont des mots presque comme les autres et il faut aussi, à ce titre, rendre justice de leur existence pour l'histoire de la revue.

Quels ont été mes points d'appui pour procéder de la sorte? J'ai eu l'occasion de m'exercer à cette manière de faire l'histoire en privilégiant intentionnellement les sources communes et anonymes que constituent deux revues séculaires portant le nom de leurs disciplines ${ }^{1}$. Avec un collègue, $j$ 'ai, avec moins de sophistication statistique, reproduit sur une plus courte période une démarche comparable pour les vingt ans de Déviance et Société (Kaminski et Cartuyvels, 1997). Ces travaux se fondent sur une seule et même hypothèse selon laquelle les mots - plus spécifiquement les mots contribuant à la formation des titres des articles publiés - constituent des sources utilisables pour une connaissance diachronique de la discipline auxquels ils appartiennent (Martindale, 1990; Wessely, 1991 ; Hogenraad et al., 1992; Hogenraad et Kaminski, 1995). Cette démarche n'est pas conçue comme une méthode stricte reproductible à l'aveugle. Elle suppose une imprégnation qualitative de la revue à laquelle elle est appliquée.

Quarante ans, pour Criminologie, c'est long. Quarante ans, pour la criminologie, c'est court. Il ne faudra donc pas attendre de mon travail une contribution à l'histoire québécoise ou montréalaise de la discipline, mais un examen des signes d'évolution interne à la revue. Afin de réaliser cette ambition modeste, j'ai créé ${ }^{2}$ et interrogé trois fichiers. Le premier fichier est le catalogue, soit l'ensemble des titres publiés entre

1. Il s'agissait de la Revue de Droit Pénal et de Criminologie et du Journal of Criminal Law and Criminology. Voir Kaminski, 1993; 1995a; 1995b; Hogenraad et Kaminski, 1995.

2. Avec l'aide précieuse d'Anne-Michèle Druetz, documentaliste au Département de criminologie et de droit pénal de l'Université catholique de Louvain. 
1968 et 2005. Ce fichier est composé de 527 références comprenant l'auteur, le titre, l'année, le volume, le numéro, le titre du numéro, la nationalité de l'auteur, la nature de la contribution (article de dossier, article hors thème, éditorial), son type formel et sa catégorisation thématique (voir infra). Le deuxième fichier est composé des thèmes des livraisons, thèmes indiqués par le titre général donné à chaque numéro (67 numéros entre 1968 et 2005; seules les sept premières livraisons ne sont pas explicitement thématiques). Le troisième fichier, qui mériterait d'être exploité plus profondément, complète significativement le précédent: il s'agit du fichier des éditoriaux de tous les numéros publiés pendant la même période. Ces textes peuvent être considérés, tout autant que les titres des articles et des numéros, comme des échantillons représentatifs du contenu des numéros.

Le présupposé transversal à l'ensemble des démarches réalisées est celui de la représentativité du contenu assurée par les mots choisis pour intituler un texte (Brown, 1964; Lindauer, 1978; Hoek, 1981; Kaminski, 1995a) ou pour représenter le thème d'un numéro de revue, par exemple.

En suivant Hoek (1981), la pertinence d'une démarche titrologique repose sur les acquis d'une conception normative, prescriptive, du titre. Autrement dit, une étude réduite au seul titre est pertinente en tant que le titre doit présenter un certain nombre de caractéristiques. Dans cette perspective, le titre doit être conforme au texte qu'il désigne: il doit marquer le texte comme il est marqué par lui. Le titre doit aussi être spécifique, autrement dit se démarquer de ses voisins. Enfin, la dimension attractive, promotionnelle, du titre ne peut être négligée.

Mais la pertinence du titre comme objet pour l'analyse peut aussi reposer sur un trait de sa description sémiotique, en l'occurrence sa semi-grammaticalité. Par semi-grammaticalité, on entend que le texte du titre se démarque de la normalité grammaticale par son style elliptique et nominal (les éléments verbaux en sont le plus souvent exclus). L'ellipse contribue à la cohérence du texte, en isolant le thème, parfois en l'isolant au point de ne distinguer en rien la nature de l'article ou la perspective qu'il prend et soutient. Cet effet est à mettre au crédit de l'usage du titre pour une analyse de contenu, alors même qu'il peut être perçu, par un regard critique, comme un signe de positivisme scientifique. Dans mon travail, je veillerai à ne pas confondre l'analyse des signes thématiques et celle de leur signification dans le champ des débats paradigmatiques qui secouent la criminologie. Cette dernière 
analyse exigerait un travail qualitatif très approfondi de l'ensemble du catalogue.

Évidemment, la conception normative et la caractéristique grammaticale des titres sont des stéréotypes. Les titres révèlent des écarts rendant au texte, par exemple, sa grammaticalité complète et d'autres encore rattachables éventuellement au domaine, au champ d'appartenance des textes étudiés. Enfin, ces «stéréotypes» n'ont de sens qu'articulés à des hypothèses sur l'évolution du discours scientifique, dont il est peu probable que quarante années suffisent à la vérification ou à la falsification. L'exercice sera donc peu démonstratif: les opérations réalisées sur la base de la consultation croisée de mes trois fichiers m'ont permis de construire un certain nombre de représentations de l'évolution de la revue, qu'il reviendra à ses animateurs anciens et actuels, à ses collaborateurs et à ses lecteurs attentifs de commenter, de rendre substantiellement significatives ou au contraire de renvoyer à l'artificialité de la méthode ou à la formalité excessive des sources de l'analyse.

Je développerai successivement certains traits quantitatifs du catalogue, des éléments de la politique éditoriale de la revue, et enfin une catégorisation thématique des objets traités par la revue.

\section{Quelques traits quantitatifs du catalogue}

Le catalogue est l'ensemble des publications de la revue (en l'occurrence 527 documents publiés entre 1968 et 2005). De nombreuses analyses seraient possibles quoique certaines resteraient peu pertinentes compte tenu du relatif jeune âge que constitue la quarantaine ici fêtée... Je me contenterai donc d'exposer quelques éléments descriptifs du catalogue et de son évolution: le nombre annuel d'articles publiés, le type formel des titres, leur colonicité, la contribution des «grands auteurs» montréalais et l'ouverture de la revue à des auteurs étrangers au Canada.

\section{L'évolution quantitative du catalogue}

De 1968 à 1976, la revue est publiée sous la forme d'un seul numéro annuel composé de cinq articles (quatre en 1972). Dès 1976, la revue publie deux numéros annuels, mais matériellement réunis en une publication (intitulée numéros 1-2), la véritable division en deux numéros ne s'opérant que l'année suivante. La revue fait plus que doubler ainsi 
son catalogue annuel. Le graphique suivant confirme la stabilisation de la composition quantitative de la revue à partir de 1977. En effet, le nombre moyen de 14 articles par an est atteint dès cette année, soit dès la première année où la revue affirme et réalise effectivement son standard de parution, inchangé depuis lors.

\section{FIG URE 1}

\section{Nombre d'articles par année}

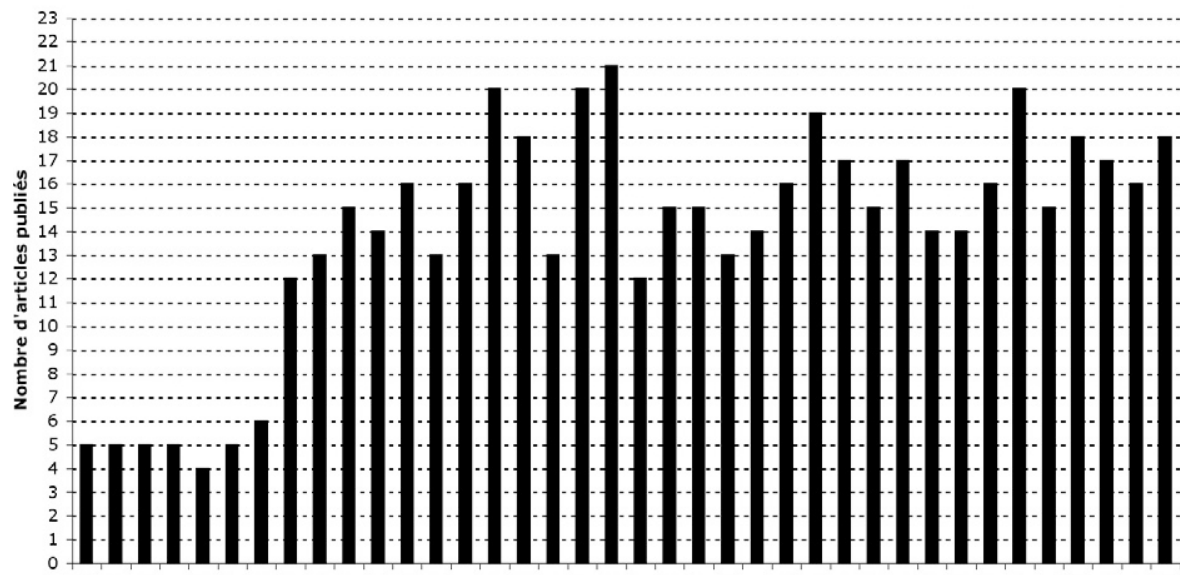

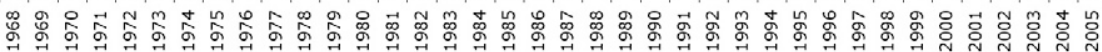
Années

\section{Le type formel des titres}

L'examen des 527 titres du catalogue permet de distinguer trois types formels de titres. Les auteurs peuvent tout d'abord choisir un titre thématique: j'entends par là que leur titre témoigne exclusivement de l'objet traité, quoi qu'il en soit de la complexité éventuelle de la formulation utilisée. Ils peuvent aussi choisir un titre problématique: dans ce cas, la forme du titre contient explicitement une question, un dilemme, un enjeu interprétatif, un mystère, qui entoure l'objet d'un halo d'incertitude. Ils peuvent enfin choisir un titre rhématique ${ }^{3}$ : dans ce cas,

3. Genette (1987: 75) distingue, dans l'usage poétique du langage, le «thème» et le «rhème», c'est-à-dire ce dont on parle et le style que l'on adopte pour en parler. Dans les usages scientifiques, cette distinction représente la distinction entre l'objet et le style de l'article, soit l'indication de sa nature: étude, réflexion, évaluation, typologie, notes, commentaires, analyse... 
l'objet de l'article est introduit et relativisé par une indication sur la nature spécifique de la contribution ${ }^{4}$; en quelque sorte l'auteur nous informe sur le type de travail qu'il a effectué sur cet objet, quitte à ce que le pléonasme affleure ${ }^{5}$. On comprendra que ces trois catégories formelles ne sont pas exclusives, dans la mesure où tout article contient une dimension thématique. Cependant, pour les besoins du codage, si un titre présente un caractère rhématique ou problématique, ces deux caractères exclusifs (entre eux) seront privilégiés sur leur caractère thématique forcément commun.

L'examen de l'évolution de la répartition de ces trois catégories de titres est un témoignage de l'évolution de l'écriture scientifique et de la complexité croissante du traitement des objets de la discipline. En quelque sorte, la dimension rhématique d'un titre fournit au lecteur le plus souvent le filtre méthodologique de l'abord de l'objet, tandis que sa dimension problématique lui fournit le filtre conceptuel, théorique ou paradigmatique de cet abord. Le titre prétend-il représenter objectivement la positivité improbable d'un objet? Souscrit-il à l'introduction du biais méthodologique ${ }^{6}$, par sa dimension rhématique, permettant au lecteur d'envisager immédiatement l'ambition scientifique de l'article ${ }^{7}$,

4. Ainsi sont toujours rhématiques les éditoriaux, les compte-rendus de symposium (s'ils sont explicitement présentés comme tels), les hommages, les errata ou encore les spécimens suivants qui contiennent une indication sur la nature de la contribution, ajoutée à l'identification de son objet: Autour de Michel Foucault et de son ouvre: Bibliographie (vol. 26, $\left.\mathrm{n}^{\circ} 1,1993\right)$ ou Entre les attentes face à la judiciarisation et l'issue des procédures: réflexion à partir d'une étude sur le traitement judiciaire des causes de violence conjugale (vol. $33, \mathrm{n}^{\circ} 2$, 2000).

5. Étude de la récidive post-traitement chez des agresseurs sexuels judiciarisés (vol. 29, $\mathrm{n}^{\circ} 1$, 1996), Étude de certains déterminants des incendies volontaires à Montréal (vol. 30, nº 1, 1997) sont deux titres exemplaires de la vacuité de certains rhèmes. Il reste que certains articles, en choisissant d'autres opérateurs rhématiques, dont la connotation scientifique est modeste, témoignent du fait que tous les articles publiés par la revue ne seraient pas des «études»: voir Notes introductives à l'étude de la criminalité des affaires (vol. 10, $\mathrm{n}^{\circ} 1,1977$ ) ou Quelques notes sur la réforme de la détermination de la peine au Canada (vol. 24, $\mathrm{n}^{\circ} 2$, 1991).

6. Le titre rhématique suivant - Comment mesurer la délinquance des toxicomanes adultes?: Une comparaison empirique de la validité des registres de police et des sondages de délinquance autoreportée (vol. $\left.35, \mathrm{n}^{\circ} 1,2002\right)$ - constitue le prototype le plus complet de la valeur substantielle du rhème, introduisant l'abord méthodologique comme filtre objectif à la positivité improbable de l'objet; il est un des rares cas dans lesquels rhème, thème et problème sont coprésents.

7. Criminalité et répression de la criminalité au Japon: analyse socio-culturelle et criminologique (vol. 14, $\mathrm{n}^{\circ} 1,1981$ ) présente une dimension rhématique (analyse) dont le service est d'assurer la scientificité par la référence à deux qualificatifs, dont l'un témoigne d'une grille de lecture et l'autre (autoréférentiel: voir infra) d'une discipline. 
la généralisation qu'il pourrait faire de ses $\operatorname{propos}^{8}$ ou sa valeur d'essai ${ }^{9}$ ? Prétend-il explicitement répondre à une question gênante ${ }^{10}$, traiter d'un dilemme ${ }^{11}$, révéler la multiplicité des perspectives ${ }^{12}$, aborder de façon critique la construction qui est habituellement faite de son objet ${ }^{13}$ ?

FIG URE 2

Type formel des titres

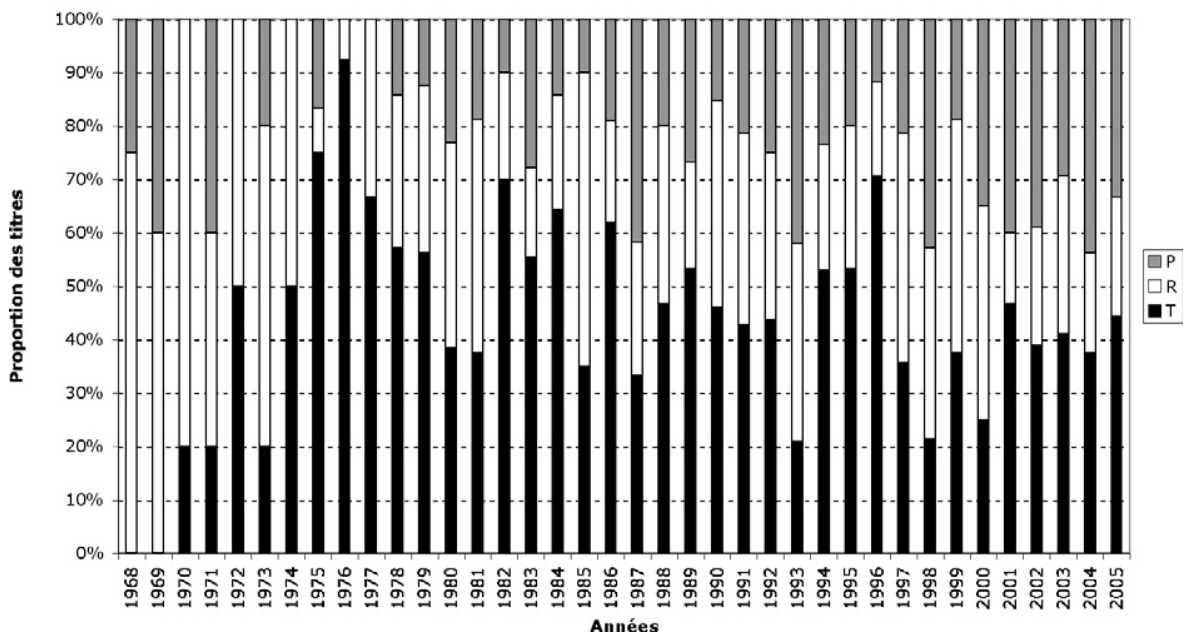

La figure 2 présente de façon empilée et proportionnelle trois courbes (intitulées: $\mathrm{T}$ pour thématique, $\mathrm{R}$ pour rhématique, $\mathrm{P}$ pour problématique) dont le tracé se confond de plus en plus au cours du temps. L'analyse porte évidemment sur des valeurs absolues assez réduites; on

8. Par exemple: De la comparution à la décision pénale, le profil judiciaire de plus de 1500 femmes (vol. 25, nº 1, 1992). En exprimant la dimension de l'échantillon, ce titre valorise un indice de scientificité de la démarche (et en particulier la valeur généralisable des résultats).

9. Ce titre: Réflexions méthodologiques pour une recherche sur l'opinion publique et l'action sociale: essai de synthèse (vol. 12, $\mathrm{n}^{\circ} 1,1979$ ) offre trois indices rhématiques successifs (réflexions, essai et synthèse). Les deux premiers témoignent d'un doute, peut-être jésuitique, sur la rigueur de la démarche et invite le lecteur à partager cette incertitude.

10. Dangerosité et justice: la peur du criminel ou la peur de la criminologie? (vol. 15, n 2 , 1982).

11. Agression et délinquance chez l'adolescente: conduites analogues ou différentes? (vol. 16, $\left.\mathrm{n}^{\circ} 2,1982\right)$.

12. Les recherches sur la police: trois approches (vol. 5, $\mathrm{n}^{\circ} 1,1972$ ).

13. Les instruments d'évaluation du risque de comportements violents: mise en perspective critique (vol. 34, nº 1, 2001). 
constate néanmoins que les titres strictement thématiques et les titres rhématiques (dans une moindre mesure) dominent le catalogue jusqu'en 1990, après quoi les courbes se confondent. Les titres dits «problématiques» manifestent une croissance lente mais tendanciellement nette sur les trente-huit années étudiées. Les titres thématiques (en noir) se maintiennent globalement autour de $40 \%$ du catalogue, les titres rhématiques (en blanc) se réduisent après les dix premières années de la revue: pendant cette période, l'affirmation rhématique aide à soutenir le positionnement de la revue dans le champ scientifique. Les titres problématiques (en gris) dépassent rarement $30 \%$ du catalogue, mais ne descendent plus en dessous de cette barre depuis le tournant du siècle.

Cette analyse porte sur les informations que le «générique» comporte ou ne comporte pas; le titre sélectionne ce qu'il met à l'affiche et ce qu'il laisse pour le récit scientifique auquel il invite. Une question spécifique relative à cette sélection concerne l'autoréférentialité (éventuelle) du titre. Il s'agit d'examiner la mesure dans laquelle les titres font explicitement référence à la criminologie dans le catalogue d'une revue qui porte justement le nom de cette discipline. Dans certaines revues, on trouve des titres significativement porteurs de ces mots que Barthes (1973) a qualifiés de «sans-gêne» et qui, en l'occurrence, répètent l'appartenance disciplinaire de la contribution. De façon cinglante, en suivant Barthes, cette forme titulaire se caractérise par sa prétention à la consistance et l'ignorance de sa propre insistance. Dans le cadre d'une revue comme la Revue de Droit Pénal et de Criminologie (voir Kaminski, 1995a), l'insistance du syntagme droit pénal dans les titres des articles «de droit pénal» relève du stéréotype et comporte la fonction explicite d'identifier la discipline du texte. Cette forme est tributaire du positivisme juridique, qui peut se contenter d'indiquer son objet et de préciser que cet objet appartient au droit pénal (dans l'hypothèse où il pourrait aussi être partagé avec d'autres branches du droit). Sur ce point, on peut avancer que l'autoréférentialité des titres du catalogue de Criminologie est faible, ce qui, on l'aura compris, est tout à son honneur. Seuls 44 titres (un peu plus de $8 \%$ du catalogue) contiennent le mot criminologie ou l'un de ses dérivés et deux usages de cette autoréférentialité se distinguent: soit l'article porte sur un événement ${ }^{14}$ ou une

14. Tel ce titre rhématique: Synthèse des travaux: premier Symposium international de recherche en criminologie comparée, Mont-Gabriel, 30 avril-3 mai 1969 (vol. 3, n 1, 1970). 
institution ${ }^{15}$ criminologique dont la référence est incontournable, soit (et ce sont les cas les plus fréquents) la discipline scientifique elle-même - dans ses crises, dans ses conflits, dans ses évolutions, dans ses formes d'institutionnalisation - constitue l'objet de l'article. À de très rares exceptions, l'auteur utilise le nom de la discipline pour signifier, de façon redondante, que son article est criminologique ou s'inscrit dans ce champ d'études.

\section{La colonicité titulaire}

Le titre scientifique manifeste des spécificités par rapport aux indications fournies ci-dessus sur la grammaticalité du titre. En particulier, un des écarts de la grammaticalité du titre scientifique se manifeste notablement par la colonicité (titular colonicity) ${ }^{16}$. Ce néologisme désigne l'usage du double point (en anglais: colon) permettant de distinguer deux membres nominaux d'un même titre. Le recours au double point est un critère formel supplémentaire susceptible d'être exploité dans le champ de la signification. L'usage du double point s'indique comme un signe de complexité, rattaché à l'érudition des textes produits dans le champ scientifique ${ }^{17}$. Il s'agit d'un indicateur de complexité dans la mesure où le double point divise une séquence tout en permettant d'articuler des concepts selon des connotations implicites. On notera d'ailleurs (et j'y reviendrai) que la colonicité d'un titre s'associe volontiers à ses dimensions rhématique ou problématique pour faire accroire à la scientificité du discours ou pour signifier la nature du questionnement sur l'objet ${ }^{18}$.

Les recherches de Dillon $(1981,1982)$ concluent que l'usage accru du double point rend compte de l'accroissement de la complexité du

15. Tel ce titre: Système de classification du Centre de documentation du Département de criminologie (vol. 1, n 1, 1968).

16. Un autre écart typiquement scientifique consiste en l'usage de la phrase grammaticale complète interrogative. En effet, la connotation de tout titre du champ étudié est de soulever un problème pour lequel le texte sert de réponse.

17. Des études de Dillon $(1981,1982)$ montrent que la grande majorité des titres publiés dans trente revues de pédagogie, de psychologie et de critique littéraire contiennent le double point, alors que ce même caractère est minoritaire dans des revues à caractère non scientifique. Voir aussi Piternick, 1991.

18. La plupart des exemples de titres ayant servi d'illustration de leur caractère rhématique ou problématique présentent aussi une division colonique. Oserais-je ajouter en exemple le titre de mon propre article? 
discours scientifique. Les revues américaines qu'il a étudiées manifestent toutes une multiplication par quatre ou cinq de l'usage du double point dans les titres des articles des trente dernières années par rapport aux années qui précèdent. Dillon faisait ce constat au début des années 1980. Les résultats, restitués graphiquement, de l'analyse de Criminologie, ne peuvent évidemment, sur ses quarante ans d'existence, répondre à l'hypothèse que Dillon a vérifiée, mais ils restent interprétables. On constate en effet une progression de la division «colonique» des titres (238 titres répondent à cette caractéristique, soit $45 \%$ du catalogue) et une réduction du recours au titre simple (le plus souvent strictement thématique).

\section{F I G URE 3}

\section{Colonicité}

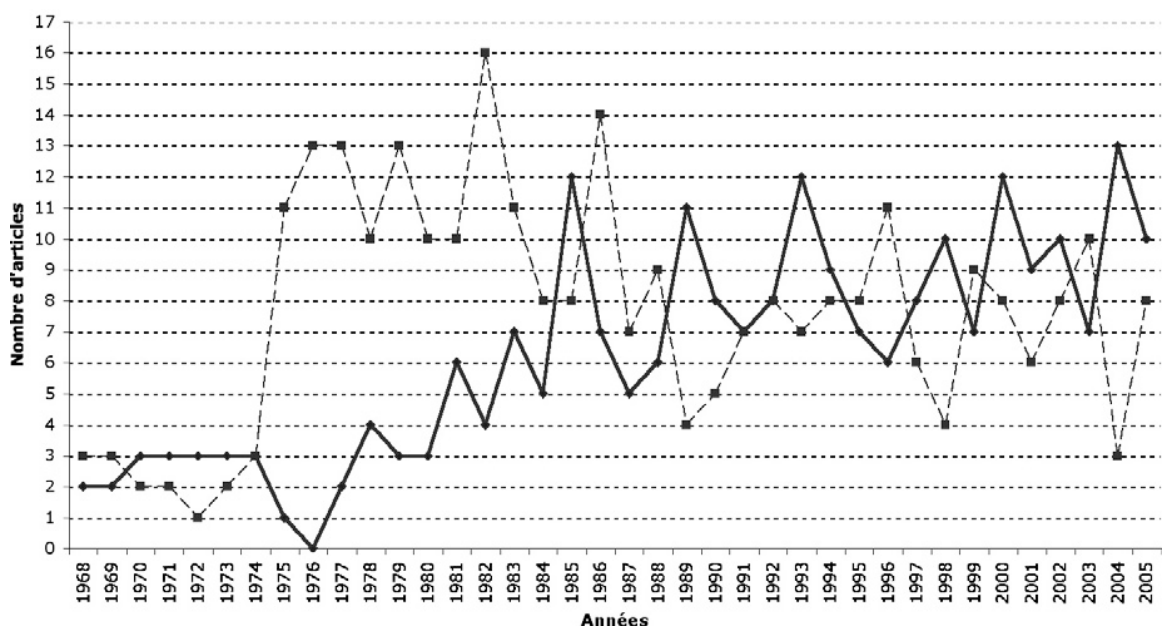

Alors que les années 1975 à 1982 sont celles de la progression du nombre total d'articles publiés, on y relève en même temps une domination manifeste du titre simple. Le renversement de tendance s'opère rapidement après sans aboutir à une domination significative du titre «colonique». Ces résultats méritent la comparaison avec la Revue (belge) de Droit Pénal et de Criminologie, dans laquelle - même en isolant la période la plus récente analysée (de 1970 à 1990) - on obtient un taux de «colonicité titulaire» maximal de $12 \%$ (Kaminski, 1995a). Dans la revue belge, ce pourcentage très réduit couvre un nombre important de 
titres dont l'usage du double point relève de la pure élégance, de l'allègement stylistique, sans le moindre effet de rétrécissement de l'objet, du point de vue ou de la perspective. Dans Criminologie, on trouve de tels usages strictement esthétiques, mais le double point prend de façon plus nette d'autres fonctions: l'association de la fonction thématique et de la fonction rhématique du titre ${ }^{19}$, le rétrécissement de l'objet (ou effet entonnoir $)^{20}$, mais aussi l'association d'une énigme poétique et de sa résolution au moins partielle ${ }^{21}$. L'usage qui parait attribuer le plus de signification substantielle au double point est celui qui distingue les deux termes du titre pour faire de l'un l'énoncé de l'objet et de l'autre l'énoncé de la problématisation de l'objet ${ }^{22}$; cet usage est relativement rare et récent dans Criminologie comme dans d'autres revues ${ }^{23}$. Cette

19. L'indemnisation des victimes d'actes criminels: étude comparative (vol. $1, \mathrm{n}^{\circ} 1,1968$ ), Violence and Robbery: A case Study (vol. 5, $\mathrm{n}^{\circ} 1,1972$ ) ou Réactions sociales à la déviance: Une étude exploratoire (vol. 7, $\mathrm{n}^{\circ} 1,1974$ ) constituent trois bons exemples de ce type de colonicité titulaire. Ils auraient tout aussi bien pu s'écrire: Étude comparative sur l'indemnisation des victimes d'actes criminels, A case Study of Violence and Robbery et Une étude exploratoire sur les réactions sociales à la déviance.

20. La police et ses auxiliaires particuliers: informateurs, délateurs et agents provocateurs (vol. 17, $\mathrm{n}^{\circ} 1,1984$ ); Enjeux de la peine et efficacité socio-juridique: le cas du braconnage (vol. 20, $\left.\mathrm{n}^{\circ} 2,1987\right)$ constituent deux exemples similaires de la fonction de réduction par l'objet. Enfance et détention correctionnelle: le cas du tribunal des jeunes de Winnipeg entre 1930 et 1959 (vol. 28, $\mathrm{n}^{\circ} 1,1995$ ) constitue un exemple de réduction par la délimitation des dimensions spatiales et temporelles de l'empirie.

21. L'attractivité du titre peut trouver sa pleine puissance dans cette fonction de la colonicité. Dans le titre suivant: Ils nont plus la liberté: réactions à la victimisation et ses conséquences (vol. 13, $\left.\mathrm{n}^{\circ} 1,1980\right)$, l'énigme est première et sa résolution partielle suit le double point. Il en va de même pour Entre l'arbre et l'écorce: la recherche criminologique au Canada (vol. 19, $\mathrm{n}^{\circ} 1,1986$ ). Dans le titre suivant: Dangerosité et justice: la peur du criminel ou la peur de la criminologie? (vol. 15, $\mathrm{n}^{\circ} 2,1982$ ), le premier membre pose l'objet de l'article et le second craint l'inquiétude poétique (tout en invitant discrètement à la problématisation). Les liaisons dangereuses: Foucault et la criminologie (vol. 26, nº 1, 1993) répond d'autant plus à cette fonction qu'il emprunte au registre littéraire.

22. On relèvera au moins deux manières de percevoir la complexification qu'introduit le double point. Le premier membre du titre peut représenter l'objet et le second la perspective dans laquelle il sera traité. Le premier terme peut être aussi celui qui est mis en doute ou à distance dans le second.

23. La couverture du crime par la presse: un portait fidèle ou déformé? (vol. 20, nº 1, 1987) et Les adolescentes membres des bandes marginales: un potentiel antisocial atténué par la dynamique de la bande? (vol. 30, $\mathrm{n}^{\circ} 1,1997$ ) constituent deux exemples d'un tel usage sous forme interrogative. L'intervention auprès de jeunes mères et de leur enfant: perspective de la théorie de l'attachement (vol. 31, $\mathrm{n}^{\circ} 2,1998$ ) ou Transformations récentes de la législation fédérale sur la mise en liberté sous condition au Canada : une lecture à la lumière des écrits sur la notion de risque (vol. 34, $\left.n^{\circ} 1,2001\right)$ illustrent le même usage par l'affichage «affirmatif» de la perspective théorique ou conceptuelle des auteurs. Vers un paradigme des inter-relations sociales? Pour une reconstruction du champ criminologique (vol. 25, $\mathrm{n}^{\circ} 2$, 1992) constitue - à l'instar du meurtre 
fonction croissante de la colonicité réduit la tradition positiviste d'un titre réduit à l'objet traité et signifie non seulement l'érudition de son auteur mais aussi son souci d'avancer son objet non comme objet décrit ou commenté, mais comme problématique ou situé dans la relativité de ses options théoriques et méthodologiques.

TA B LEA U 1

Colonicité et type formel des titres

\begin{tabular}{|l|c|c|c|c|c|c|c|c|}
\hline $\begin{array}{c}\text { Type formel } \\
\text { Colonicité }\end{array}$ & \multicolumn{3}{|c|}{ Thématique } & \multicolumn{2}{c|}{ Rhématique } & \multicolumn{2}{c|}{ Problématique } & \multicolumn{2}{c|}{ Total } \\
\hline & N & $\%$ & N & $\%$ & N & $\%$ & N & $\%$ \\
\hline Titre simple & 171 & 70 & 86 & 53,5 & 32 & 26,5 & 289 & 55 \\
\hline Titre colonique & 74 & 30 & 75 & 46,5 & 89 & 73,5 & 238 & 45 \\
\hline Total & 245 & 100 & 161 & 100 & 121 & 100 & 527 & 100 \\
\hline
\end{tabular}

On peut observer quantitativement, dans ce tableau, l'association entre la colonicité et les caractères rhématique ou problématique du titre. Le titre thématique est le plus souvent simple ( $70 \%$ des occurrences), le titre rhématique reste simple avec une courte majorité $(53,5 \%)$ et le titre problématique renverse complètement la tendance avec une structuration colonique majoritaire $(73,5 \%)$. Autrement dit, les accroissements respectifs de la problématicité des titres et de leur colonicité constituent conjointement les indices d'une tendance normale à la complexification du discours scientifique tenu par la revue.

\section{La part des grands auteurs montréalais}

Les chercheurs de l'École de criminologie ou du Centre international de criminologie comparée (CICC) de l'Université de Montréal règnent évidemment sur la production de la revue. La qualification de «grands auteurs» ne doit pas tromper: elle est destinée à représenter une vingtaine de chercheurs de Montréal dont la contribution à la revue dépasse quatre articles et ne peut aucunement servir à disqualifier les chercheurs qui choisissent de publier les résultats de leurs recherches dans d'autres

sans cadavre - un exemple de colonicité sans double point. Le point d'interrogation joue le rôle de séparateur de deux éléments, l'un consacré à l'objet, l'autre à la perspective. On notera, à cet égard, que seuls 39 titres du catalogue $(7,5 \%)$ usent du point d'interrogation. 
organes. La proportion de ces "grands auteurs» est historiquement décroissante. Le graphique suivant présente en noir le poids de leur contribution. Il ne souligne aucunement le déclin de la performance de ces chercheurs; il permet plutôt de visualiser l'accroissement historique du réservoir d'auteurs. Le calcul opéré privilégie donc les auteurs dont la longévité est à ce jour suffisante pour révéler cette ouverture de la revue aux «autres» auteurs, au fur et à mesure de sa propre croissance.

F I G URE 4

Contributions des «grands auteurs»

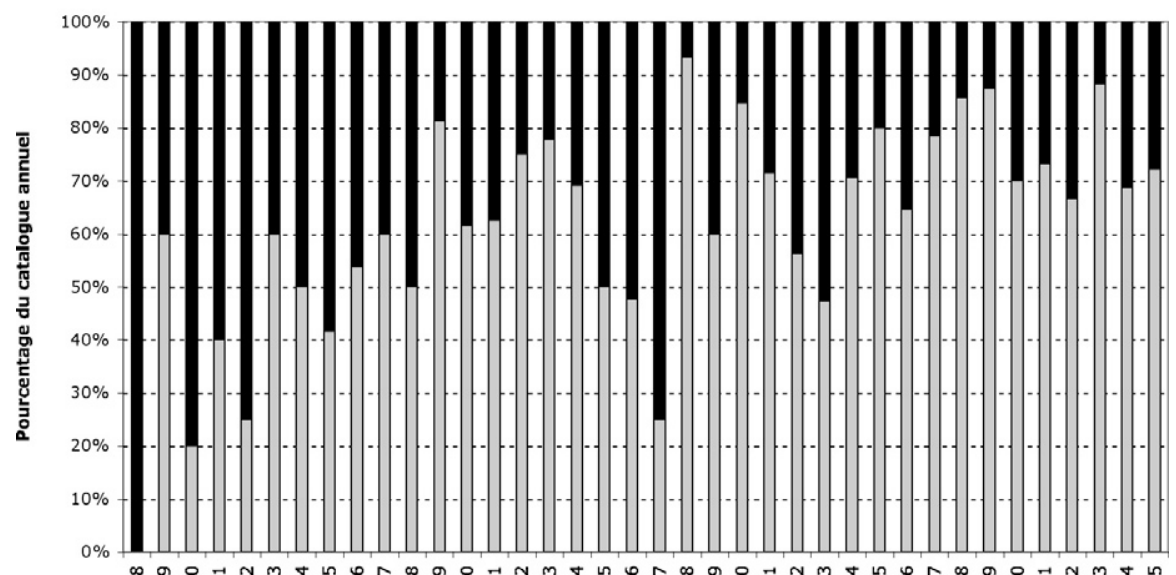

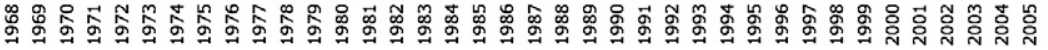
Années

Parmi ces grands auteurs, on citera Marc LeBlanc avec 22 articles, Maurice Cusson, Alice Parizeau, André Normandeau et Pierre Landreville avec 14 articles, Denis Szabo avec 13 contributions (parmi lesquelles sept éditoriaux), Jean-Paul Brodeur avec 12 publications. Les autres auteurs comptabilisés ont moins de dix publications à leur actif; 184 contributions sont ainsi attribuées, soit $35 \%$ du catalogue complet de la revue.

Si l'on envisage maintenant les directions de numéros thématiques explicitement reconnues par la revue, Pierre Landreville détient le record de la direction avec cinq numéros, suivi de Jean-Paul Brodeur qui en a dirigé quatre. Jean Trépanier et Pierre Tremblay ont assuré chacun la direction de trois numéros. Guy Lemire, André Normandeau, Sylvie Frigon, Alvaro Pires, Marc LeBlanc, Mylène Jaccoud, Jean 
Poupart, Marie-Marthe Cousineau, Jean Proulx, Louise Biron et Maurice Cusson ont chacun assuré la direction de deux numéros. Nous savons qu'il ne faut pas confondre les deux unités de compte que sont les crimes et leurs auteurs (parce qu'un auteur peut avoir commis plusieurs crimes et qu'un crime peut avoir plusieurs auteurs); c'est ainsi que les chiffres suivants doivent bien être considérés comme une statistique des «directeurs» et non des numéros dirigés: les 15 noms déjà évoqués assurent 32 directions de numéros, sur les 45 attribuées nominativement. Treize numéros thématiques sont donc attribués, hors cas de codirection avec l'un des auteurs déjà cités, à des auteurs n'ayant dirigé à eux seuls ou avec un autre codirecteur non encore cité qu'un seul numéro (Fernando Acosta, Sheilagh Hodgins, Serge Brochu, Jean Lajoie, May Clarkson, Lucie Lemonde, Marc Ouimet, Jean-Luc Bacher, Carmen Poulin, Gilles Rondeau, André Cellard, Michel Janosz, Arlène Gaudrault, Marc Daigle, Nadine Lanctôt). Rappelons que les 14 premiers numéros ne font pas l'objet d'une direction nominativement reconnue.

\section{Les contributions étrangères}

Seule revue de criminologie publiée en français sur le continent américain (comme le rappelle la présentation qui figure en page 2 de chaque numéro), la revue se concentre bien évidemment sur les recherches québécoises ou issues de l'environnement immédiat de la province. Sur le catalogue de 527 articles, 65 (12,5\%) sont signés par des auteurs étrangers au Canada (il ne s'agit pas ici de prendre en compte la nationalité de l'auteur, mais bien son affectation professionnelle sur le plan géographique).

Ces auteurs viennent de France (22 occurrences), de Belgique (16 occurrences) des États-Unis (12 occurrences), de Grande-Bretagne (cinq occurrences), les dix dernières publications étrangères venant d'Allemagne, du Japon, d'Italie, de Nouvelle-Zélande, de Slovénie, des PaysBas, de Suisse.

La progression du recours aux auteurs étrangers est significative. Le nombre de contributions étrangères (au Canada) s'accroît avec l'âge de la revue et avec le souci manifeste d'ouvrir ses colonnes à la francophonie européenne essentiellement. En 2000, les proportions habituelles sont inversées, mais c'est en raison de la publication des actes du symposium international de victimologie tenu à Montréal. La figure 5 
FIG URE 5

Auteurs canadiens et étrangers

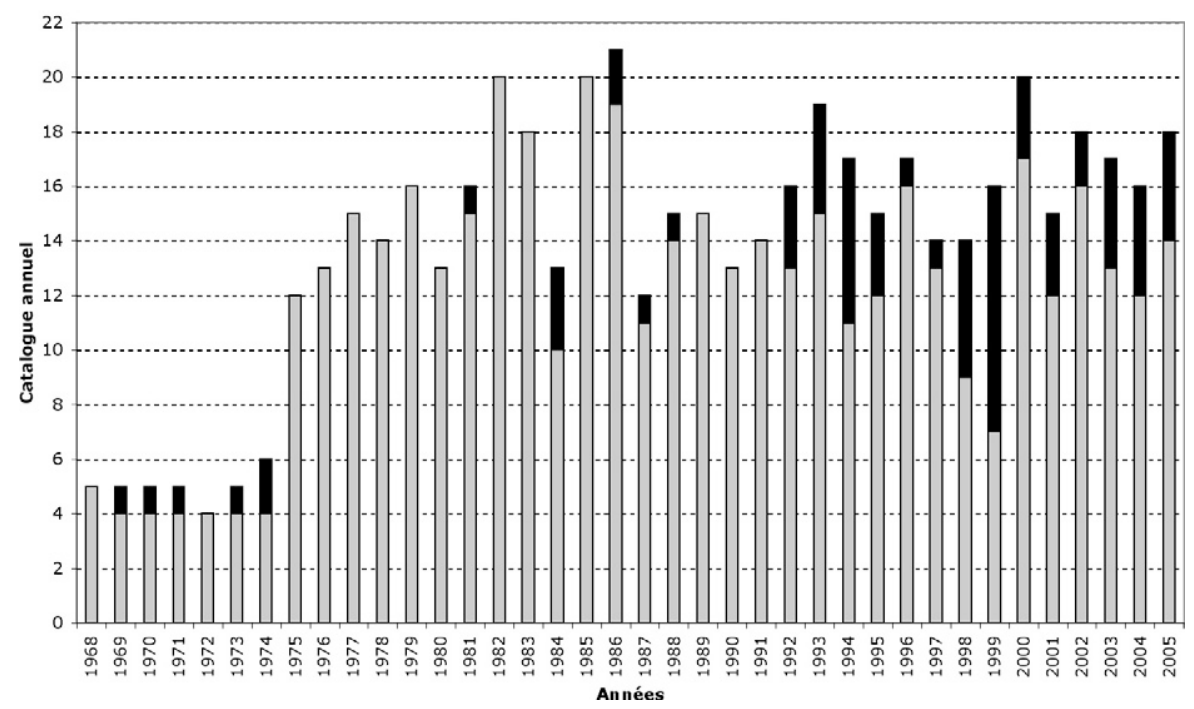

visualise la part quantitative (relativement faible) de ces productions étrangères (en noir) dans le catalogue annuel de la revue. Tant en nombre absolu d'articles qu'en proportion, 1999 constitue donc l'année la plus internationale de Criminologie.

\section{La politique éditoriale}

La revue naît et grandit sur l'établissement et l'explicitation d'un projet qui a subi des mutations successives. Ces mutations ne font pas varier un trait transversal et significatif de l'identité de la revue: la publication systématique d'un éditorial, véritable passeport du numéro (en période normale) ou de la revue (en période de crise). Les éditoriaux seront les supports empiriques de l'analyse qui suit.

L'histoire lisible de l'adoption d'une structuration thématique

Je présenterai dans les lignes qui suivent les inflexions formelles successives prises par le comité de direction, volontairement ou non, pour rendre compte de l'orientation de la revue. Très jeune, la revue 
Criminologie a décidé de se donner une composition thématique ${ }^{24}$. Dès le volume 8 (1975), la revue prend explicitement cette tournure: un thème oriente dorénavant la composition des numéros. Les 39 articles dits «hors thème» représentent néanmoins 7,5\% du catalogue.

Le volume $16 n^{\circ} 2$ (1983) est le premier à faire place à la mise en exergue du nom de la personne qui a dirigé la production du numéro. Serait-ce parce qu'il traitait d'un thème que le comité éditorial ne pouvait assurer lui-même (Les femmes et la justice pénale)? Le comité de rédaction signe encore anonymement un éditorial jusqu'en 1984 (volume $17, \mathrm{n}^{\circ} 1$ ), puis semble confier au(x) directeur(s) du numéro son (leur) autonomie jusque dans la conception de l'éditorial, modèle inchangé depuis le numéro 2 de $1986^{25}$. Pierre Robert signe par contre l'introduction du numéro qu'il coordonne avec Pierre Tremblay sur Le milieu criminel (vol. 22, n 2, 1989), mais le texte est consacré à signaler un tournant éditorial de la revue (Éditorial: La nouvelle formule de Criminologie). Dans le numéro suivant, l'éditorial est pour la première fois titré, par Jean Lajoie, de façon significative (Éditorial: Adolescence et désespoir du sens) eu égard au thème du numéro. À l'exception du numéro suivant, cette méthode de titrage de l'éditorial devint la règle.

À partir du volume $28 \mathrm{n}^{\circ} 1$, l'éditorial se transforme en présentation du numéro. Le volume 29 revient cependant à l'appellation Editorial et s'y ajoute l'évocation d'une saison de parution (printemps et automne), mais, dès le volume $29 \mathrm{n}^{\circ} 2$ de 1996, l'éditorial reprend sa structuration «colonique», le titre générique étant suivi d'un double point donnant accès aux termes significatifs de l'orientation du numéro. En 1999, il n'y a plus de saisons; l'appellation et le style du texte introductif varieront de façon apparemment erratique ${ }^{26}$. En tout cas à partir du volume

24. Une analyse qui ne tiendrait pas compte de cette structuration ne pourrait être qu'illusionnée sur la nature du catalogue de la revue. Autrement dit, on ne peut analyser le catalogue à l'aveugle comme un agencement arbitraire mais non aléatoire de titres, comme je l'ai fait notamment pour deux revues séculaires et non thématiques comme la Revue de Droit Pénal et de Criminologie et le Journal of Criminal Law and Criminology.

25. On balbutie encore un peu entre 1984 et 1986 puisque l'éditorial du volume 18 $\mathrm{n}^{\circ} 2$ (1985) n'est pas signé du nom d'André Normandeau, alors qu'il dirige ce numéro sur Le vol à main armée à Montréal.

26. À partir de 1999, à deux reprises, la direction d'un numéro est confiée à un «couple international» (Mylène Jaccoud et Lode Walgrave; Arlène Gaudrault et Tony Peters). Le volume $33 n^{\circ} 2$ est particulier à plus d'un titre: Le Mot du directeur (Pierre Landreville) qui l'introduit en témoigne: document produit dans le cadre d'un symposium (Dixième symposium de victimologie) et distribué à ses participants, ce numéro contient des textes en anglais et en français (chose qui ne s'était plus présentée depuis les origines de la revue). 
35, toute référence à l'introduction du numéro disparaît au profit d'un titre (identique au thème annoncé ou différent de celui-ci) signé par le directeur du numéro. Depuis 1984, les numéros sont «préparés» par leur responsable (jusqu'en 1986, puis à nouveau en 1989). L'année suivante, les éditoriaux ne sont plus signés par personne. Et dès le numéro 2 de 1986, c'est le responsable du numéro qui signe l'éditorial, tradition inchangée depuis lors.

\section{Des Acta Criminologica à Criminologie}

Initialement publiée sous le nom d'Acta Criminologica et sous-titrée Études sur la conduite antisociale, la revue Criminologie révèle quelques crises de croissance, avant qu'elle n'acquière sa vitesse et sa tonalité de croisière. Il vaut le détour d'examiner les symptômes encore lisibles de ces crises normales. Jusqu'en 1975, la revue publie des textes en anglais et «appartient» incontestablement à son fondateur et directeur, qui signe personnellement les éditoriaux, consacrés à la fois à la présentation du contenu du volume et à l'affirmation de considérations théoriques rendant compte du projet scientifique de la revue. Trois préoccupations sont reflétées par la criminologie, selon Denis Szabo, dans l'éditorial du premier volume (1968) : l'intégration théorique, la mesure du phénomène criminel et l'application des connaissances scientifiques à l'administration de la justice. Mais le contenu du volume étant une illustration de chacune de ces préoccupations, il est difficile de savoir si celles-ci sont générales et exclusives d'autres soucis ou si elles ont été construites pour les besoins de la présentation des contributions du volume. On penche pour la deuxième hypothèse lorsqu'on lit les éditoriaux successifs des volumes suivants.

Si l'on en fait une lecture un peu attentive, la nature des éditoriaux change perceptiblement avec le temps. Le volume 8 (1975) constitue une quadruple rupture sur le plan formel: on a déjà vu que la revue devient explicitement thématique à partir de cette date; elle acquiert dès l'année suivante un nouveau rythme de parution (deux numéros annuels encore confondus en 1976); la revue cesse de publier des articles en anglais, ce qu'elle avait fait jusques et y compris dans son volume 7; enfin disparaît la signature du fondateur éditorialiste Denis Szabo. Cette disparition, à cet endroit, du nom du fondateur de la revue semble devoir être associée (temporellement sinon causalement) à une 
transformation plus radicale de la politique de la revue. Jusqu'en 1975, chaque éditorial est l'occasion de l'expression d'un credo criminologique ou d'un défi, ce qui sied évidemment au lancement d'une nouvelle revue, qui plus est, celle qui réunit les criminologues de langue française en Amérique, autant dire les illustres habitants d'un village gaulois. Les credos s'accumulent au fil des sept premières années et s'amenuisent dans le même temps. La recherche d'une "synthèse théorique nouvelle» (vol. 1), la participation d'une production québécoise et montréalaise à l'enrichissement de la «connaissance universelle» (vol. 1), le souci de faire reconnaître la multidisciplinarité comme «un signe de richesse» (vol. 2) ou encore de valoriser la recherche empirique car le «tribunal implacable des faits [est] le seul qui compte pour l'homme de science» (vol. 2), le plaidoyer pour «un pluralisme des méthodes et un éclectisme théorique» (vol. 3) afin que, de la comparaison et de l'analyse transculturelle, émerge la mort du relativisme (vol. 3), toutes ces expressions intégratrices et unificatrices saturent les premiers volumes.

Alors qu'une rupture majeure se manifeste en 1975, des évolutions plus minuscules se manifestent entre 1968 et cette date. Ainsi, les deux premières livraisons étaient rapidement saturées d'études, de monographies variant entre 60 et 100 pages et constituaient aussi une vitrine de la jeune école de criminologie de Montréal. Dans le même esprit, le volume 1 publie le système de classification du centre de documentation du département de criminologie de l'Université de Montréal et le volume 2 fournit un document exposant longuement le programme de cours et de recherches du même département. En 1969, le Centre international de criminologie comparée (CICC) est fondé et la revue Acta Criminologica s'offre en caisse de résonance des symposiums du CICC (voir vol. 3). En 1973, l'éditorialiste, conscient de la nature de la revue, propose un changement de formule qui n'aura guère d'avenir, appuyé cependant sur un constat qui sera le moteur du changement produit en 1975 : la multiplication des recherches et des voies de publication. Le désir de privilégier la criminologie comparée y est réaffirmé.

Les volumes 4 et 5 font cependant place à des enjeux politiques et théoriques qui ne peuvent qu'émousser la confiance à la fois scientiste et unitariste des premiers éditoriaux. Pour la première fois en 1971, Denis Szabo constate «une transformation des intérêts des criminologues depuis quelques années» (vol. 4) et ouvre les préoccupations à celle de la sélection inégalitaire des conduites et des personnes par les 
appareils de justice pénale. En effet, «les recherches portent de plus en plus sur l'appareil social créé pour combattre le crime» (vol. 4) et l'on constate que «la "sélection" des conduites ainsi que celle des personnes qualifiées de "délinquantes" ne se fait point au hasard» (vol. 4). Ce double constat se radicalise dans la dénonciation du «caractère artificiel et même arbitraire de la définition de la criminalité» (vol. 5) et Denis Szabo y ajoute la production d'un «véritable cul-de-sac de la théorie du comportement [...] produit à cause de la prétention de certains théoriciens de tenter d'expliquer la criminalité comme phénomène indifférencié » (vol. 5). La confiance intégrative des premiers éditoriaux est ici érodée. Le volume 6 témoigne de la persistance d'un espoir pour le moins fendu: «espérons que la plus grande diversité du présent volume ne constitue pas une dispersion indue [...]. Elle se veut le reflet de la diversité croissante [...] des recherches criminologiques» (vol. 6). La pertinence des travaux et l'amélioration de la condition humaine constituent deux nouvelles formulations des préoccupations scientifiques et éthiques de la revue, formulations plus modestes que les préoccupations exprimées en 1968. L'éditorial du volume 7 (1974) s'ouvre sur la persistance de la tension de la discipline exprimée en des termes divers : la «double face de la criminalité», le «caractère provisoire des résultats», la mise «en question de la démarche étiologique de la criminologie», la «crise de légitimité» du "pouvoir politique dans les démocraties occidentales». L'éditorial se ferme sur une nouvelle affirmation du défi criminologique et de la vertu comparatiste; l'espoir est cette fois clairement indexé à un postulat cartésien, touchant dans la modestie de sa formulation: "sans le développement des études dans ce domaine toutefois [la criminologie comparatiste], il serait vain d'espérer une plus profonde compréhension de notre système de justice comme de notre propre criminalité» (vol. 7).

Cette longue évocation des éditoriaux de Denis Szabo signe la réduction progressive des ambitions de la discipline criminologique. Paradoxalement cette réduction semble avoir été le point de passage obligé pour une réorientation dont la pertinence est prouvée par sa persistance. À l'invocation de la vanité conditionnelle de l'espoir criminologique, correspond la mort des Acta Criminologica. La naissance de Criminologie (entraînant la disparition du sous-titre des Acta) se fonde sur une invocation de type nouveau: «Depuis 1967, le monde a changé», nous apprend l'éditorialiste anonyme du volume 8 (1975). 
Deux exigences s'expriment: le resserrement sur les travaux produits au Québec ${ }^{27}$ et la diffusion de leurs résultats au public professionnel et cultivé (le nombre de criminologues québécois croissant par ailleurs). Un fil éthique apparaît inchangé malgré les transformations auxquelles je ferai rapidement allusion dans les lignes qui suivent. En effet Denis Szabo attribuait à la criminologie le «souci permanent d'améliorer la condition humaine» (vol. 6). On trouve dans l'éditorial anonyme du volume $10 \mathrm{n}^{\circ} 2$ (1977) consacré à la criminologie au Québec une invocation sensiblement comparable de la «recherche d'une société plus juste où il fait bon vivre».

\section{Les conséquences de la refonte de 1975}

Trois types de conséquences - formelles, institutionnelles et fondamentales - découlent de la naissance de Criminologie.

Formellement, le respect des nouvelles exigences entraîne la publication d'articles plus brefs (les longues monographies des premières années paraissant dorénavant sous forme de livres) et la diversification des rubriques. Cette diversification constitue le dernier cahot formel de la revue avant le nouveau dispositif simplifié, éprouvé sans discontinuer depuis 1989 (un dossier thématique et des articles hors thème). On notera que les articles dits «hors thème» ne sont explicitement présentés sous cette appellation qu'à partir de 1993.

L'éditorialiste du volume 8 (1975) révèle aussi l'institutionnalisation de la revue. Il annonce ainsi le développement ultérieur des collaborations nécessaires à l'administration de la vie autonome d'un périodique. La structure de la revue s'est étoffée, dès 1975, d'une secrétaire de rédaction, Alice Parizeau (remplacée par Jacqueline De Plaen en 1983), et d'un directeur adjoint en la personne d'André Normandeau. Le comité de rédaction reste cependant composé des trois membres recrutés depuis la naissance de la revue (Henri Ellenberger, Marcel Fréchette et José M. Rico). En 1979, Jacques Laplante entre au comité pour en ressortir en 1983 et y revenir en 1984 avec son affiliation à l'Université d'Ottawa et en compagnie de Pierre Landreville. Jean Trépanier y entre

27. En conséquence de quoi, la revue ne fera plus place à la langue anglaise, à l'exception notable (et explicitement justifiée par Pierre Landreville, directeur de la revue) du numéro spécial (2000) préparé pour le $\mathrm{X}^{\mathrm{e}}$ symposium international de victimologie. 
en 1980. Le comité s'accroît et surtout se diversifie en 1983 en s'adjoignant des nouveaux collaborateurs venant d'autres universités québécoises (UQAM, Sherbrooke notamment, puis Ottawa). En 1987, André Normandeau devient le directeur de la revue et Denis Szabo rejoint un conseil d'administration (auquel il n'avait jamais été fait allusion jusquelà). La liste des correspondants internationaux disparaît pendant que le comité de rédaction se réduit à Fernando Acosta, Serge Brochu, JeanPaul Brodeur et Sheilagh Hodgins. En 1989, Serge Brochu reprend la direction jusqu'en 1997, date à laquelle Pierre Landreville le remplace jusqu'en 2005. Dianne Casoni est la directrice actuelle. Ces différents signes de mobilité (auxquels une histoire moins formelle que celle que je fais ici donnerait une signification substantielle) me permettent de rendre compte de trois caractéristiques d'une institution: l'autonomie acquise par la revue, la transmission de sa direction aux générations successives et sa puissance fédératrice.

Plus fondamentalement, la reconnaissance de la diversification des recherches et de l'enrichissement de la criminologie québécoise (sur laquelle la revue décide de se centrer) prolonge les constats antérieurs de Denis Szabo, mais contrarie dorénavant le projet soutenu (jusque-là) de valoriser la démarche comparative. Cette reconnaissance implique aussi l'attribution d'une valeur positive et centrale aux accrocs que le fondateur de la revue voyait s'agrandir sur la toile de son projet unificateur. En effet, si le volume 8 (1975) ne révèle pas de rupture autre que formelle dans la construction de la revue, la présentation du thème - la délinquance juvénile au Québec - fait cependant place à des énoncés significatifs: "on a découvert l'influence décisive de la législation sur la définition même de la conduite délictueuse [...] Les criminologues se penchent donc sur les lois» (vol. 8). C'est dans l'éditorial de 1981 (vol. 13, no 1) se penchant sur le tournant de 1975 que le comité présente la politique éditoriale suivie depuis lors comme marquée par la visée de «présenter aux lecteurs une analyse systématique de la réaction sociale à la criminalité, essentiellement au Québec» (vol. 13, n 1). La revue cherche, dès 1975, à témoigner «d'une idéologie pluraliste, d'un caractère scientifique, en un mot, de la diversité des travailleurs intellectuels et professionnels de notre milieu» (vol. 8, 1975). On trouve ici la reconnaissance explicite d'une préoccupation pluraliste (et non plus intégrative) et diversificatrice (plutôt qu'unificatrice). On ne peut exprimer plus violemment le tournant épistémologique que l'éditorialiste 
anonyme du volume 9 (1976): «Enfin, que dit la "science", dont l'autorité est aussi contestée aujourd'hui qu'elle fut naïvement adulée il y a dix ans seulement?» (vol. 9, 1976). On peut dire que la nouvelle formule, tant dans ses exigences fondamentales que dans ses conséquences pragmatiques et formelles, restera fondamentalement inchangée dans les trente années suivantes. L'éditorial de 1982 réaffirme son souci de se recentrer sur les recherches québécoises et de représenter les diverses tendances de la criminologie. La question des tendances, des courants, des épistémologies va parcourir ainsi toute l'histoire de la revue, sous des présentations elles-mêmes diversifiées ${ }^{28}$.

\section{Une revue thématique}

\section{Le rangement des extravagances}

L'histoire de toutes les revues est traversée par des soucis de rangement de ses «extravagances». Elles résolvent chacune à leur manière le problème de l'excédent, du hors normes, par la création de rubriques spéciales dans lesquelles ranger des contributions échappant aux standards qu'elles se sont définis.

Les rubriques en question sont, jusqu'en 1993, des témoignages, des notes de lecture, des informations, des actualités, des notes de recherche et des entretiens. Le graphique ci-dessus en fournit la distribution. La table de données qui figure sous le graphique proprement dit permet de visualiser la distribution (étendue, mais aux effectifs réduits) des différentes rubriques. Avec les articles dits hors thème (officiellement annoncés en 1989 dans l'éditorial du volume 22, n 2 et explicitement

28. Par exemple, dans l'éditorial du volume 17, $\mathrm{n}^{\circ} 1$ (1984), Jean-Paul Brodeur distinguera les recherches instrumentales et les recherches critiques (sur la police) auxquelles il sera fait place dans le numéro qu'il dirige; dans l'éditorial du volume 18, ${ }^{\circ} 1$ (1985), le souci de travailler l'histoire est relié à la crise des années soixante qui a vu se produire «l'éclatement de l'objet criminologique»; de même, le numéro spécial du $25^{\mathrm{e}}$ anniversaire de l'École de criminologie de l'Université de Montréal manifeste on ne peut mieux les clivages persistants et reconnus; en 1992 (vol. 25 n 2), Alvaro Pires consacre un dossier sur le renouveau en criminologie et l'introduit par ces mots: «L'euphorie et la vitalité critique des années soixante-dix dans le champ de la criminologie a cédé la place au marasme et à la cacophonie des années quatre-vingt. Où en sommes-nous à l'aube de la dernière décennie du $\mathrm{xx}^{\mathrm{e}}$ siècle? Perçoit-on les signes de nouvelles articulations sur le plan théorique ou encore entre les savoirs et les pratiques? Comment la criminologie se placerat-elle face aux débats qui l'ont déchirée?». 
FIGURE 6

Rubriques spéciales

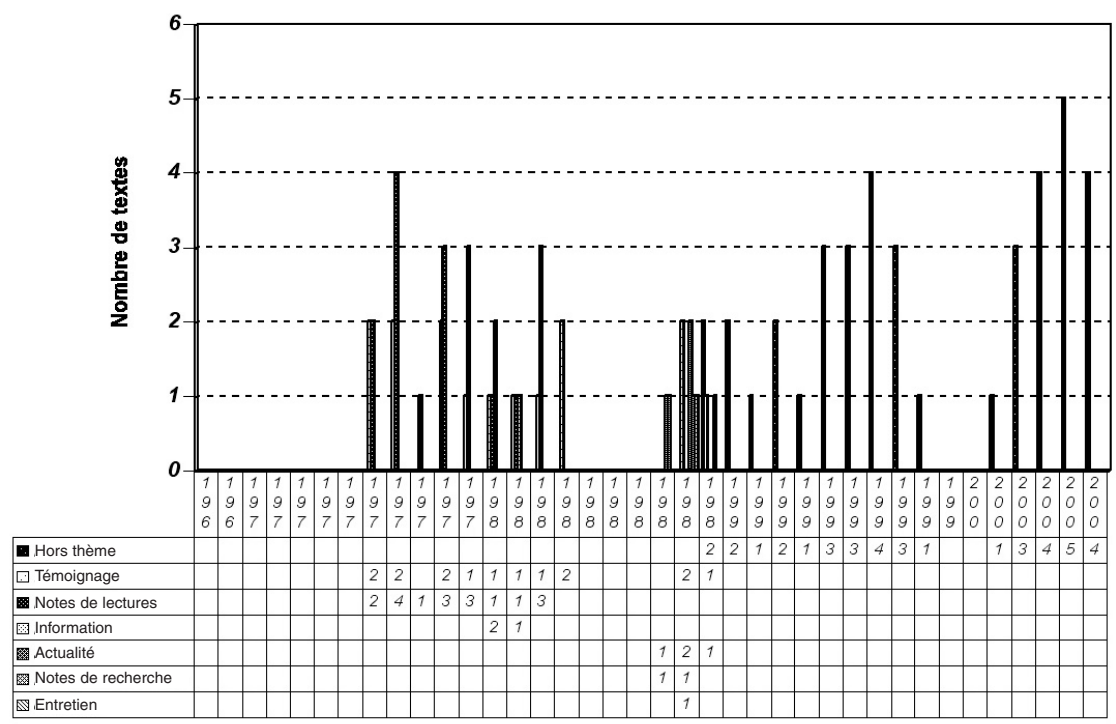

présentés sous cette appellation à partir de 1994), 75 articles peuvent ainsi être réunis dans ces rubriques spéciales. Nombreuses et diversifiées pendant les vingt premières années de vie de la revue, dès lors qu'elle a atteint son catalogue de croisière, après cette période d'adolescence, la revue a simplifié sa structuration en organisant, sous cette seule catégorie, la publication d'articles «hors thème», soit des articles qui échappent à la thématique du numéro.

L'année 1988 constitue le comble de la multiplication de ces rubriques. Mais on y décèle un paradoxe: les rubriques en question sont tout sauf hors thème et désignent simplement des inflexions quant à la nature de la contribution sur le thème du numéro. Au contraire, la catégorie «hors thème» ne désigne aucunement la qualité particulière de la contribution. Elle concerne 39 articles, soit 7,5\% du catalogue de la revue.

On visualisera dans le graphique ci-dessous la proportion des rubriques spéciales (en noir) année par année. 
FIGURE 7

Proportion des rubriques spéciales

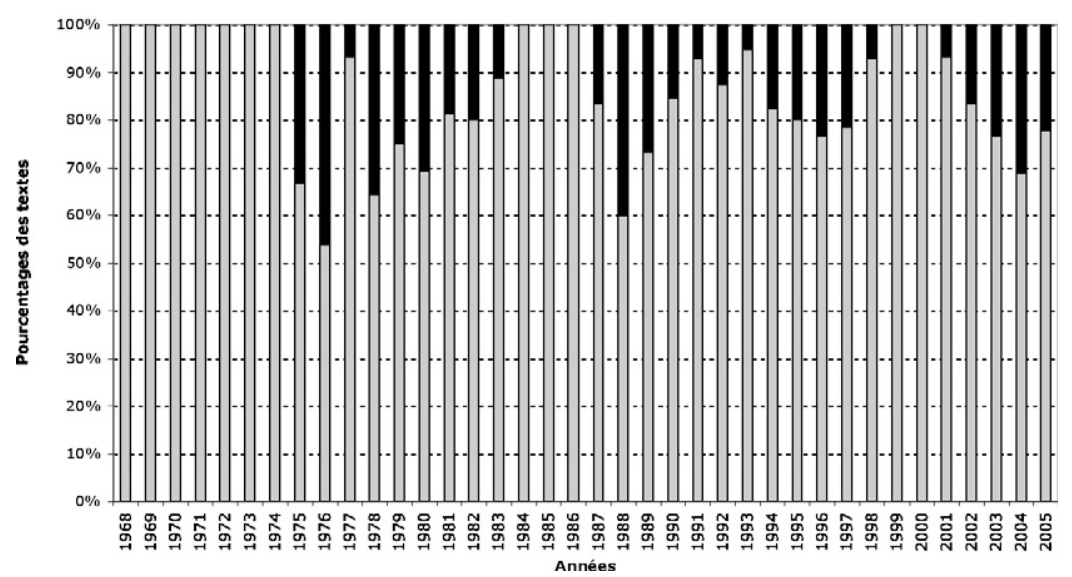

Les catégories thématiques

Une méthode - trop simple, certes - de catégorisation du catalogue de la revue consiste à se fier à la structuration thématique qui, dès le volume 8 (1975), caractérise Criminologie jusqu'à aujourd'hui. Pour ce faire, je me suis permis, à l'épreuve de l'imprégnation des titres et en particulier des éditoriaux des 67 numéros traités, de distinguer quatre grandes catégories représentant, à des degrés différents, les objets. Non exclusives (j’y reviendrai), ces catégories témoignent de manières différentes d'indiquer le domaine du projet spécifique du numéro. Ainsi, un thème peut attirer l'attention sur une population spécifique, sur une forme de délinquance, sur un dispositif de contrôle ou sur une discipline scientifique. Certains croisements entre ces catégories sont évidemment autorisés par le codage et effectivement observés dans la revue. Je confronterai les résultats quantitatifs de cette méthode avec le codage du catalogue de la revue selon les mêmes catégories.

Les populations, soit des sous-populations visées dans le titre du numéro ou son éditorial, sont les jeunes (six numéros surtout concentrés au début de la période de publication), les femmes (six numéros dont quatre sont distribués dans les dix dernières années de la période analysée), les victimes (quatre numéros répartis de façon extrêmement équilibrée), et de façon réduite, les malades mentaux (deux numéros) et les minorités ethniques (un numéro). Dix-neuf numéros sont, à titre 
principal, marqués par le traitement d'une question relative à l'une ou l'autre de ces populations. Le codage des 527 articles du catalogue de la revue fournit 169 entrées (32\%) se référant explicitement à l'une ou l'autre de ces sous-populations spécifiques. Dans le catalogue apparaissent de façon quantitativement peu significative deux autres populations spécifiques (les auteurs d'infractions à caractère sexuel et les usagers de drogues). En tout cas, de façon dominante, la problématique de l'adolescence (masculine et féminine) et des femmes, tant victimes qu'auteures, saturent les thématiques de numéros et le catalogue d'articles consacrés à des populations.

La délinquance et ses formes diversifiées sont l'objet manifeste de 25 livraisons sur les 67 analysées. Par délinquance, j'entends ici tout signifiant relatif à toute forme d'infraction pénale, à son auteur ou à sa comptabilisation $^{29}$. La catégorie peut cependant être répartie en quatre sous-catégories: parfois une figure criminelle est favorisée (le vol à main armée à Montréal, la délinquance des affaires); la délinquance est parfois attachée à une population évoquée ci-dessus (la délinquance juvénile, les violences conjugales et les homicides conjugaux); est parfois privilégiée l'articulation causale ou phénoménologique entre la délinquance et ses facteurs (drogues et criminalité, analyse spatiale du crime, suicide et délinquance, l'âge et la question criminelle); enfin une technique de connaissance ou d'action est parfois indexée à la délinquance (intervention, prédiction, prévention). Aussi, 138 entrées (soit $26 \%)$ du catalogue répondent à la même définition.

Les dispositifs de contrôle dominent expressément la production de 34 numéros. Par contrôle, j'entends ici tout signifiant renvoyant à une modalité technique, institutionnelle ou politique de connaissance et de gestion de la délinquance pour autant qu'elle soit l'objet «interrogé» du numéro. Il s'agit, sur le plan institutionnel, des sous-systèmes de l'administration de la justice pénale que sont la police, les tribunaux et la prison, ainsi que leurs fonctions ou outils spécifiques (la prévention policière, la libération conditionnelle, la détention préventive...); il s'agit aussi des formes de régulation que constituent le droit (ou les droits) et les politiques criminelles et pénales, ou encore la sécurité privée; il s'agit enfin de concepts théoriques et pratiques transversaux à l'aune desquels se construisent les dispositifs de contrôle (dangerosité,

29. On reconnaîtra ici les trois niveaux d'interprétation (crime, criminel, criminalité) en criminologie. 
insécurité, risque). La répartition des 34 numéros, eu égard aux 25 numéros consacrés à la délinquance, mérite une observation. Dans les dix premières années, la thématique de la délinquance et celle de son contrôle sont «confondues», probablement en raison de la diversité thématique des premiers numéros. Ces deux catégories sont ensuite le plus souvent dissociées, se succédant sans se recouvrir. On notera que les thématiques du contrôle s'articulent plus volontiers avec l'identification d'une population (par exemple l'enfermement des femmes) qu'avec une problématique délinquante. Dans le catalogue, 264 articles, soit exactement $50 \%$, se rangent dans la catégorie des dispositifs de contrôle.

Ainsi la revue donne, à l'analyse, l'image d'une forte polarisation, sans point de rencontre, entre les objets d'une criminologie axée sur la connaissance de la délinquance et ceux d'une criminologie axée sur la connaissance des dispositifs de gestion de la délinquance.

Enfin, des numéros de la revue sont explicitement consacrés à la mise en exergue d'une perspective disciplinaire ou d'une interrogation sur la discipline. Il peut s'agir de la criminologie, de l'histoire, de la psychiatrie, disciplines au sens strict à laquelle on associera aussi la méthodologie comparative, spécifiquement valorisée à Montréal. Quinze numéros sont affectés explicitement soit au traitement disciplinaire d'une thématique spécifique $^{30}$ soit à la problématisation de la discipline criminologique ${ }^{31}$. Le début des années 1990 est particulièrement saturé de ces productions. Le codage des articles permet de dévaluer l'importance quantitative de cette référence disciplinaire: il ne couvre en effet que 42 articles, soit $8 \%$ du catalogue.

Le tableau suivant fournit la distribution comparée des quatre catégories appliquées successivement aux thématiques de numéros et au catalogue complet de la revue.

30. Par exemple, Libération conditionnelle: évolution et application (1899-1981), paru en 1981, dans le volume $14 \mathrm{n}^{\circ} 2$ ou Le construit de l'ethnicité en criminologie, paru en 2003, dans le volume $36 \mathrm{n}^{\circ} 2$, ou encore L'bistoire de l'enfermement et des populations pénales, paru en 1995, dans le volume $28 \mathrm{n}^{\circ} 1$.

31. Nouvelles connaissances et nouvelles questions en criminologie paru en 1992 (vol. $\left.25 n^{\circ} 2\right)$ ou Criminologie: discipline et institutionnalisation. Trois exemples francophones, paru en 2004 (vol. $37 \mathrm{n}^{\circ} 1$ ). 
TABLEAU 2

Distribution des catégories thématiques

\begin{tabular}{|l|c|c|c|c|}
\hline & \multicolumn{2}{|c|}{$\begin{array}{c}\text { Thématiques } \\
\text { des numéros }\end{array}$} & \multicolumn{2}{c|}{$\begin{array}{c}\text { Catalogue } \\
\text { de la revue }\end{array}$} \\
\hline & $\mathrm{N}$ & $\%$ & $\mathrm{~N}$ & $\%$ \\
\hline Population & 19 & 28 & 169 & 32 \\
\hline Délinquance & 25 & 37 & 138 & 26 \\
\hline Contrôle & 34 & 50 & 264 & 50 \\
\hline Discipline & 15 & 22 & 42 & 8 \\
\hline
\end{tabular}

Je rappellerai ici que les catégories ne sont pas exclusives, raison pour laquelle les totaux ne peuvent être pris en compte et les pourcentages dépassent le $100 \%$. Le recouvrement ne concerne en fait que deux cas de figure quantitativement significatifs: celui de la population et de la délinquance (53 articles, soit $10 \%$ du catalogue) d'une part, et celui de la population et du contrôle (98 articles, soit 18,5\% du catalogue) d'autre part. Le tableau comparatif présenté ci-dessus permet de croire que l'intégration des thématiques de la délinquance et de son contrôle est plus grande que le donne à croire le titre des numéros de la revue et se prolonge au-delà des dix premières années. Les articles officiellement considérés comme «hors thème» (39 articles, soit 7,5\% du catalogue) sont évidemment susceptibles de contribuer à cette apparente intégration.

FIGURE 8

Distribution des catégories thématiques

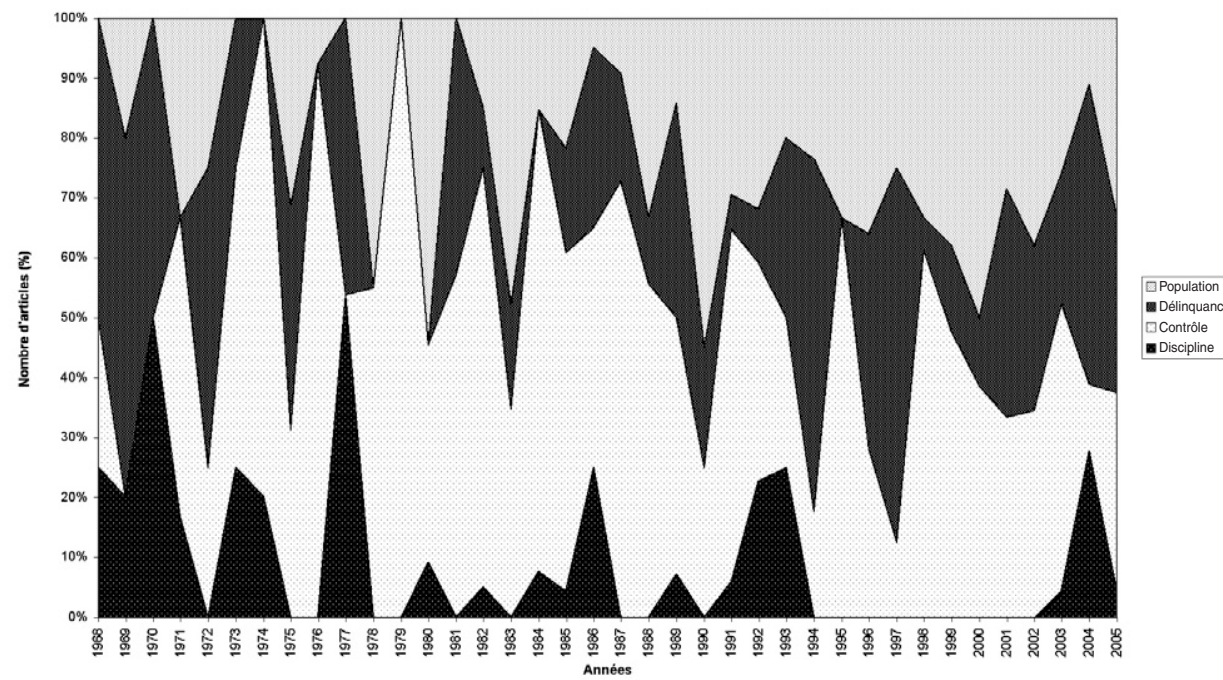


Le graphique ci-dessus représente la part contributive (en pourcentages) ${ }^{32}$ des catégories thématiques dans le catalogue (sachant que, compte tenu de la non exclusivité des catégories, les pourcentages ne renvoient pas au nombre réel d'articles). Il importe de ne pas confondre la catégorisation thématique opérée ici avec une catégorisation paradigmatique: les textes consacrés à un dispositif de contrôle ne sont pas nécessairement constructivistes ou critiques, tout comme les textes consacrés au phénomène délinquant ne relèvent pas nécessairement d'une criminologie positiviste. Jean-Paul Brodeur, dans son éditorial de 1984 (volume $17, \mathrm{n}^{\circ} 1$ ) distingue bien, à propos de la police, une double orientation paradigmatique, représentée en l'occurrence dans le numéro qu'il dirige: une criminologie «instrumentale» y côtoie une criminologie qui (sans être qualifiée de façon aussi cinglante) privilégie «l'analyse du rôle de la police dans la "fabrication" de la criminalité». De même, le traitement de la criminalité des affaires au Québec (1977, volume 10, n 1) diffère sensiblement sur le plan paradigmatique du numéro de 1988 (volume 21, $\mathrm{n}^{\circ} 1$ ) intitulé Entre les mailles de la loi: pollution, accident de travail, faute professionnelle; leurs objets réfèrent néanmoins à des problématiques comportementales codées comme telles dans le traitement que j'inflige ici à la revue. Si l'on revient à la distribution comparée du tableau ci-dessus, on peut donc affirmer que le traitement d'une thématique délinquante peut s'opérer en recourant (explicitement) à l'analyse de dispositifs de contrôle; l'inverse n'est cependant pas vrai...

TABLEAU 3

Catégories thématiques et type formel des titres

\begin{tabular}{|l|c|c|c|c|c|c|c|c|}
\hline $\begin{array}{l}\text { Type formel } \\
\begin{array}{l}\text { Catégorie } \\
\text { thématique }\end{array}\end{array}$ & \multicolumn{2}{|c|}{ Thématique } & \multicolumn{2}{l|}{ Rhématique } & \multicolumn{2}{l|}{ Problématique } & \multicolumn{2}{c|}{ Total } \\
\hline & $\mathrm{N}$ & $\%$ & $\mathrm{~N}$ & $\%$ & $\mathrm{~N}$ & $\%$ & $\mathrm{~N}$ & $\%$ \\
\hline Délinquance & 65 & 47 & 38 & 27,5 & 35 & 25,5 & 138 & 100 \\
\hline Contrôle & 152 & 57,5 & 45 & 17 & 67 & 25,5 & 264 & 100 \\
\hline Total & 217 & 54 & 83 & 20,5 & 102 & 25,5 & 402 & 100 \\
\hline
\end{tabular}

Un dernier exercice peut encore être entrepris sur la base du tableau présenté ci-dessus. Trouve-t-on une corrélation entre la distinction

32. Une représentation en courbes non cumulées fournirait une image des plus confuses. 
proposée entre les deux principales catégories thématiques et le style formel - thématique, rhématique et problématique - des titres?

Assurément, la progression des titres dits problématiques ne se distingue aucunement selon le thème (délinquance ou contrôle) auxquels ils sont consacrés. Par contre, on constate que la dimension rhématique du titre est plus importante lorsque son objet est axé sur la délinquance et que la dimension thématique du titre est plus importante lorsque son objet est axé sur le contrôle.

\section{Conclusion}

Il n'y a pas de conclusion. Mais la formule s'impose pour loger discrètement - honteuse complaisance - les vœux de longue vie d'un auteur à la revue qui le publie...

\section{Références}

Barthes, R. (1973). Le plaisir du texte. Paris: Seuil.

Brown, W. P. (1964). The Titles of Paperback Books. British Journal of Psycho$\log y, 55$ (3), 365-368.

Dillon, J. T. (1981). The Emergence of the Colon: An Empirical Correlate of Scholarship. American Psychologist, 36 (8), 879-884.

Dillon, J. T. (1982). In pursuit of the Colon: A Century of Scholarly Progress, 1880-1980. Journal of Higher Education, 53 (1), 91-97.

Genette, G. (1987). Seuils. Paris: Seuil.

Hoek, L. H. (1981). La marque du titre. Dispositifs sémiotiques d'une pratique textuelle. La Haye-Paris-New York: Mouton.

Hogenraad, R., Bestgen, Y., \& Durieux, J.-F. (1992). Psychology as Literature. Genetic, Social, and General Psychology Monographs, 118, 457-478.

Hogenraad, R., \& Kaminski, D. (1995). Trails of Science: The Visibility of Scientific Progress in Criminology. Social Science Information, 34 (4), 663685.

Kaminski, D. (1993). Le titre du savoir, étude titrologique de la Revue de droit pénal et de criminologie. Revue de Droit Pénal et de Criminologie, avril, 386425.

Kaminski, D. (1995a). Entre criminologie et droit pénal. Un siècle de publications en Europe et aux États-Unis. Bruxelles: Presses de l'Université de Montréal, Presses de l'Université d'Ottawa, De Boeck Université.

Kaminski, D. (1995b). Une histoire à lire entre les mots: les mouvements de la criminologie. Déviance et Société, 19 (4), 307-323.

Kaminski, D., \& Cartuyvels, Y. (1997). Vingt ans et toutes ses dents? Déviance et Société, 21 (1), 77-95. 
Lindauer, M. S. (1978). Physiognomic Meanings in the Titles of Short Stories. In C. Martindale (ed.), Psychological Approaches to the Study of Literary Narratives (74-95). Hamburg: Helmut Buske Verlag.

Martindale, C. (1990). The Clockwork Muse. The Predictability of Artistic Change, New York: Basic Books.

Piternick, A. B. (1991). From Incipits to Colon Counts: a Natural History of Titles. Scholarly Publishing, 22 (3), 170-183.

Wessely, A. (1991). Transposing "Style" from the History of Art to the History of Science. Science in Context, 4 (2), 265-278. 